\title{
Synthesis of Some Novel Compounds of Saccharinyl Acetic Acid Containing Nucleus and Evaluation of Their Biological Activities as Antimicrobial
}

\author{
MAGDA H. ABDELLATTIF* \\ Pharmaceutical Chemistry Department, Pharmacy College, Deanship of \\ Scientific Research, Taif University, Taif, KSA. \\ ${ }^{*}$ Corresponding author E-mail: magdah11uk@ hotmail.com \\ http://dx.doi.org/10.13005/ojc/320164
}

(Received: January 22, 2016; Accepted: March 06, 2016)

\begin{abstract}
A new series of Compounds of Saccharinyl Acetic acid Containing nucleus have been prepared via an improved synthetic procedure. Where saccharinyl moiety have been introduced to 4-benzylidine-2-methyl-1,3-oxazole-5-one in position 2 , compound (3) which has been reacted with nitrogen neucleophiles as hydrazine hydrate, phenyl haydrazine, aniline, $p$-toludine, $m, p$-aminobezoic acid to get compounds from (4-6). Also the reaction of compound (3) witharomatic substrate in presence of anhydrous $\mathrm{AlCl}_{3}$ (Friedel - Crafts reaction) afforded acetamide derivative (7) via the elimination of arylidine group. Moreover saccharinyl acetic acid hydrazide (8) was refluxed in acetic anhydride to give benzisothiazole derivative (9), which reacted with carbon nuleophiles (Grignard reagent) to afford compound (10). But when compound (9) reacted with $\mathrm{PCl}_{5} / \mathrm{POCl}_{3}$ it gave compound (11) which reacted with urea and thiourea to give compound (12(a, and b)). Also the condensation of compound (9) with aromatic aldehyde gave compound (13). Structures of all synthesized compounds were elucidated from I.R., 'HNMR, mass-spectroscopy, and elemental analysis.
\end{abstract}

Key words: Saccharin, hydrazinehydrate, oxazole, imidazole, benzisothiazole.

\section{INTRODUCTION}

The reported pharmaceutical properties of saccharin and its derivatives ${ }^{1-4}$ and in connection ${ }^{5-9}$ with our ongoing interest to developing new synthetic strategies for construction of heterocyclic systems involving saccharin due to its significant biological ${ }^{10}$. ${ }^{12}$ and pharmacological activities. Also imidazole derivatives are of interest to medical chemists for many years because of their biological activities such as anticancer, anti tubercular, antibacterial, and antifungal activities ${ }^{13-22}$ In view of the above facts we report in the present work the synthesis and antimicrobial activities of Saccharinylderivatives.

\section{METHODS AND EXPERIMENTS}

\section{Chemistry}

Melting points were determined in open capillaries using Gallen Kamp melting point 
apparatus and are uncorrected F.T.-IR spectra $(\mathrm{KBr}$. disc) were recorded on a Perkin Elmer 1720 F.T. - IR spectrometer. ${ }^{1} \mathrm{H}-\mathrm{NMR}$ spectra were recorded on varin Gemini NMR spectrometer $300 \mathrm{MHz}$ using TMS as internal standard. All reactions were monitored by TLC using aluminum silica gel plates 60 F 245 . Elemental analysis and antimicrobial activity were carried out at micro-analytical center, faculty of science, cairo university, Egypt. Elemental analysis of all synthesized compounds is in agreement with the structure elucidated.

\section{4-Benzylidene-2-Saccharinyl methyl-1,3-Oxazole- 5-one.(3)}

A mixture of Saccharinyl acetic acid chloride $(10 \mathrm{mmol})$ and glycine $(10 \mathrm{mmol})$ were refluxed in boiling ethanol for $1 \mathrm{hr}$. The spectral solid was then refluxed for $4 \mathrm{hr}$. in freshly distilled acetic anhydride $(30 \mathrm{ml})$ and anhydrous sodium acetate in the presence of benzaldehyde. The reaction mixture was poured onto water and solid that separated was re-crystallized from ethanol to give (3). Orange (68\%) mp 140-142 ${ }^{\circ} \mathrm{C}$, IR (KBr, $\left.\mathrm{Cm}^{-1}\right)$ : v 1700 (10 azlactone), $1610(\mathrm{CN}), 1337$ and $1120\left(\mathrm{SO}_{2}\right)$. El - MS: m/z $368\left[\mathrm{M}^{+}\right]$. Analytical calculation for $\mathrm{C}_{18} \mathrm{H}_{12} \mathrm{O}_{5} \mathrm{~N}_{2} \mathrm{~S}$; C58.7; H3.3; N7.6 found C58.65; H3.3; $\mathrm{N}, 7.55^{28,30}$

5-Benzylidene-3-saccharinyl methyl-1, 2, 4-triazin-6-one $(4 a, 4 b)$

A mixture of $3(10 \mathrm{mmol})$ and hydrazine hydrate or phenyl hydrazine ( $10 \mathrm{~mm}$ ol) in absolute ethanol $(30 \mathrm{ml})$ was refluxed for $3 \mathrm{hr}$. the solid that obtained was recrystallized from ethanol to afford 4a: orange ( $75 \%)$, mp $163-165^{\circ} \mathrm{C}$; IR $\left(\mathrm{KBr}, \mathrm{cm}^{-1}\right): 1700$ (CO) , $1615(\mathrm{C}=\mathrm{N})$, 3350-3450 (br NH - NH) and 1337 and $1138\left(\mathrm{SO}_{2}\right) \mathrm{MF} \mathrm{C}_{18} \mathrm{H}_{14} \mathrm{O}_{4} \mathrm{~N}_{4}$.S EI $-\mathrm{MS}$ : $\mathrm{m} / \mathrm{z} 382\left(\mathrm{M}^{+}\right)$. And 4b : brown ( 80\%), mp 95 -97; IR $\left(\mathrm{KBr}, \mathrm{cm}^{-1}\right): 1680$ ( CO) , 1610 ( C=2), $3250(\mathrm{NH})$, 1330 and $1168\left(\mathrm{SO}_{2}\right) \mathrm{MF} \mathrm{C}_{24} \mathrm{H}_{18} \mathrm{O}_{4} \mathrm{~N}_{4} \mathrm{~S}$. El- MS: m/z $460\left(\mathrm{M}^{+}\right)$

General procedure for the preparation of cinnamamide derivatives ( $5 a-d$ ) and imidazole one derivatives (6a-d).

A mixture of $3(10 \mathrm{mml} \mathrm{ol})$ and aromatic amine oraminobenzoicacid) was reflexed for $3 \mathrm{~h}$. in absolute ethanol (30 ml). For compounds (5a-d) and in n-nutanol $(30 \mathrm{ml})$ for compounds $(6 \mathrm{a}-\mathrm{d})$. The solid that obtained was recrystallized from proper solvent to afford (5a-d) and / or (6a-d). ${ }^{30}$

\section{( $\alpha$-Saccharinyl- $\mathrm{N}$ - acetamido) cinnamamide} (5a)

Yellowish (70\%), mp $210-212$; IR( KBr, $\mathrm{cm}^{-1}$ ) : 1670 and 1660 (CO), 3300-3200 (NH), 1330 and $1170\left(\mathrm{SO}_{2}\right)$. MF $\mathrm{C}_{24} \mathrm{H}_{19} \mathrm{O}_{5} \mathrm{~N}_{3} \mathrm{~S}^{\prime} \mathrm{El}-\mathrm{MS}: \mathrm{m} / \mathrm{z}$ $461\left(\mathrm{M}^{+}\right)$, crystallized from benzene $(5 \mathrm{~b})$ dark brown (75\%) , mp 219-221; IR( $\left.\mathrm{KBr} \mathrm{cm}^{-1}\right) .1670$ (CO), 3320, $3250(\mathrm{NH}) 1616(\mathrm{C}=\mathrm{N}), 1320$ and $1170\left(\mathrm{SO}_{2}\right)$.

MF $\mathrm{C}_{25} \mathrm{H}_{21} \mathrm{O}_{5} \mathrm{~N}_{9} \mathrm{~S}$, El- MS: m/z $476(\mathrm{M}+1)$ recrystallized from toluene.

(5c) yellow (65\%) ,mp 90-92, IR(5Br, cm 1):1720 , (CO) , 1680 , (CO amide), 3350-3400 (broud $\mathrm{NH}, \mathrm{OH}), 1310$ and $1120\left(\mathrm{SO}_{2}\right) \cdot \mathrm{MF} \mathrm{C}_{25} \mathrm{H}_{19} \mathrm{O}_{7} \mathrm{~N}_{3} \mathrm{~S}, \mathrm{EI}$ -MS: m/z $505\left(\mathrm{M}^{+}\right)$, crystallized from benzene.

(5d) brown ( 70\%) , mp 110-112, IR ( $\mathrm{KBr}, \mathrm{cm}^{-1}$ ):1710 (CO), 1670 ( CO amide) , 33003450 (broud $\mathrm{NH}, \mathrm{OH}), 1300$ and $1110\left(\mathrm{SO}_{2}\right)$.

MF $\mathrm{C}_{25} \mathrm{H}_{19} \mathrm{O}_{7} \mathrm{~N}_{3} \mathrm{~S}$. El - MS : m/z $505\left(\mathrm{M}^{+}\right)$. recrystallized from benzene.

(6a) brown (75\%), mp 180-182, IR( $\mathrm{KBr}$, $\mathrm{cm}^{-1}$ ):1660 (CO) , 1600 ( CO amidazolone) 1580 $(\mathrm{C}=\mathrm{N}) .1330$ and $1160\left(\mathrm{SO}_{2}\right) . \mathrm{MF} \mathrm{C}_{24} \mathrm{H}_{17} \mathrm{O}_{4} \mathrm{~N}_{3} \mathrm{~S}$ El- MS : m/z $443\left(\mathrm{M}^{+}\right)$recrystallized from toluene.

(6b). brown (65\%) ,mp 202-204, IR( $\left.\mathrm{KBr}, \mathrm{cm}^{-1}\right)$ :

1670 (CO), 1630 ( CO imidazole), 1580( C=N), 1320 and $1170\left(\mathrm{SO}_{2}\right)$. M.F. $\mathrm{C}_{25} \mathrm{H}_{19} \mathrm{O}_{4} \mathrm{~N}_{3} \mathrm{~S}$. El- MS: $\mathrm{m} / \mathrm{z} 457\left(\mathrm{M}^{+}\right)$.

(6c) brown (70\%), mp 150-152, IR ( $\mathrm{KBr}$, $\left.\mathrm{cm}^{-1}\right), 1680$ (coimidazolone) , $1610(\mathrm{C}=\mathrm{N}), 1320$ and $1110\left(\mathrm{SO}_{2}\right), 3450(\mathrm{OH}) . \mathrm{MF} \mathrm{C}_{25} \mathrm{H}_{17} \mathrm{O}_{6} \mathrm{~N}_{3} \mathrm{~S}$ El-MS: $\mathrm{m} / \mathrm{z} 487\left(\mathrm{M}^{+}\right)$, recrystallized from benzene.

(6d) yellowish (77\%) ,mp 155-157, IR ( $\mathrm{KBr}$, $\left.\mathrm{cm}^{-1}\right)$ : 1690 (Coimidazolone) , $1620(\mathrm{C}=\mathrm{N}), 1280$ and $1120\left(\mathrm{SO}_{2}\right) 3440(\mathrm{OH})$.

M.F $\mathrm{C}_{25} \mathrm{H}_{17} \mathrm{O}_{6} \mathrm{~N}_{3}$ S. El- MS : m/z $487\left(\mathrm{M}^{+}\right)$ recrystallized from toluene. 
General procedure for the preparation of imidazolone derivatives (6a-d) From ( saccharinyl$\mathrm{N}$ - acetamido ) cinnamide derivatives( $5 \mathrm{a}-\mathrm{d}$ ) by cyclization .

A solution of compounds $5 \mathrm{a}-\mathrm{d}$ ( $10 \mathrm{~m}$ mole) in acetic anhydride $(15 \mathrm{ml})$ was boiled under reflux for $2 \mathrm{~h}$. The resulting solution was poured onto crushed ice, and the product that separated out was filtered off, washed with solution of sodium hydrogen carbonate followed by water and then dried. The products were recrystallized from a proper solvent.

\section{Saccharinyl (N'- benzoyl methyl) acetamide(7)}

A solution of oxazolonederivative $3(10 \mathrm{~m}$ mole) in dry benzene was treated with anhydrous $\mathrm{Alll}_{3}(30 \mathrm{~m}$ mole) with continuously stirring on water bath for $3 \mathrm{~h}$.

The reaction mixture was decomposed with ice- cold hydrochloric acid. Then the ethereal layer was separated and dried over anhydrous $\mathrm{Na}_{2} \mathrm{SO}_{4}$.
The excess solvent was evaporated then the separated compound was recrystallized from benzene to give (7) : buff (60\%) , mp 115-117, IR $\left(\mathrm{KBr}, \mathrm{cm}^{-1}\right), 1700$ (CO ketone), 1680 (CO imide), $3340(\mathrm{NH}), 1330$ and $1140\left(\mathrm{SO}_{2}\right) \mathrm{MF} \mathrm{C}_{17} \mathrm{H}_{14} \mathrm{O}_{5} \mathrm{~N}_{2} \mathrm{~S}$. El-MS: $\mathrm{m} / \mathrm{z} 358\left(\mathrm{M}^{+}\right)$

6- Oxo -1, 2,4 - triazino $(4,3-b)(1,2)$ benzoisothiazole(9)

A mixture of compound 8 ( $10 \mathrm{~m}$ mole) in redistilled acetic anhydride $20 \mathrm{ml}$ was refluxed for $1 \mathrm{~h}$. the reaction mixture was cooled and poured onto ice - cold water. The solid product separated was filtered and recrystallized from ethanol to afford compound (9), yellowish brown (65\%) , mp 145-147, IR ( $\mathrm{KBr}$, $\left.\mathrm{cm}^{-1}\right): 1710(\mathrm{CO}), 3432(\mathrm{OH}), 3100(\mathrm{NH}) 1634$ $(\mathrm{C}=\mathrm{N}) 1363$ and $1133\left(\mathrm{SO}_{2}\right)$, HNMR $\left(\right.$ DMSo-d $\left._{6}\right) \mathrm{S}$ : $2.5\left(\mathrm{~s}, 2 \mathrm{H}, \mathrm{cH}_{2}\right), 4.4(\mathrm{~s}, 1 \mathrm{H}, \mathrm{NH}), 5.7(\mathrm{~s} .1 \mathrm{H}, \mathrm{OH}$ of lactam- lactim dynamic equilibrium) and 7.8-8.1 (m, $4 \mathrm{H}, \mathrm{ArH}), \mathrm{MF} \mathrm{C}_{9} \mathrm{H}_{7} \mathrm{~N}_{3} \mathrm{O}_{3} \mathrm{~S}, \mathrm{El}-\mathrm{ms}, \mathrm{m} / \mathrm{z} 251\left(\mathrm{M}^{+}\right)$.

Table 1: Antimicrobial activity (in vitro) of some synthesized compounds

\begin{tabular}{|c|c|c|c|c|}
\hline \multirow[t]{2}{*}{ Compd no. } & \multicolumn{4}{|c|}{ Zone of inhibition $\mathrm{mm} / \mathrm{mg}$ of sample } \\
\hline & $\begin{array}{l}\text { colickca } \\
\text { coli }\end{array}$ & $\begin{array}{c}\text { Staphylococcus } \\
\text { aureus }\end{array}$ & $\begin{array}{l}\text { Candida } \\
\text { albicans }\end{array}$ & $\begin{array}{l}\text { Aspergillus } \\
\text { flavus }\end{array}$ \\
\hline 3 & 0 & 0 & 17 & 15 \\
\hline $4 a$ & 0 & 0 & 15 & 0 \\
\hline $4 b$ & 18 & 12 & 15 & 18 \\
\hline $5 a$ & 0 & 0 & 15 & 0 \\
\hline $5 b$ & 0 & 0 & 16 & 0 \\
\hline $5 g$ & 0 & 0 & 16 & 0 \\
\hline $5 d$ & 12 & 14 & 0 & 0 \\
\hline $6 c$ & 16 & 12 & 15 & 0 \\
\hline 7 & 15 & 10 & 15 & 0 \\
\hline 9 & 0 & 0 & 10 & 0 \\
\hline $10 a$ & 19 & 0 & 10 & 0 \\
\hline 11 & 0 & 0 & 15 & 0 \\
\hline $12 b$ & 0 & 0 & 0 & 0 \\
\hline $13 a$ & 18 & 0 & 0 & 0 \\
\hline Tetracycline & 30 & 28 & 0 & 0 \\
\hline Amphotericin & n 0 & 0 & 16 & 20 \\
\hline
\end{tabular}


General procedure for the preparation of 6 alkyl-6-hydroxyl- 1,2,4- triazino $(4,3-b)(1,2)$ benzisothiazole (10a-c).

To Mg metal ( $10 \mathrm{~m}$ mole) in dry ether $(40 \mathrm{ml})$ an alkyl halide namely, ethyl iodide, methyl iodide or benzyl chloride (30m mole) in dry ether $(20 \mathrm{ml})$ was added dropwisely. The reaction mixture was refluxed and compound a (10m mole) in dry ether $(40 \mathrm{ml})$ added portion wise within $1 \mathrm{~h}$.

The reaction mixture was further refluxed for $3 \mathrm{~h}$, left over night and then decomposed with dil cold $\mathrm{HCl}$. The ethereal layer was / washed with $\mathrm{NaHCO}_{3}$ solution then water and dried over analydrous $\mathrm{Na} 2$ $\mathrm{SO}_{4}$ and evaporated to give compound 10a-c which was recrystallized form benzene (10a) : ball yellow (6690) , mp (195-197, IR( KB , cm $\left.^{-1}\right)$ ) 3420 -3390 $(\mathrm{OH}$ and $\mathrm{NH}), 1610(\mathrm{C}=\mathrm{N}), 1310$ and $1130\left(\mathrm{SO}_{2}\right)$ , MF C $\mathrm{C}_{10} \mathrm{H}_{11} \mathrm{~N}_{3} \mathrm{O}_{3} \mathrm{~S}$, El -MS: $\mathrm{m} / \mathrm{z} 3\left(\mathrm{M}^{+}\right)$.

(10b) : yellowish (70\%), mp 172-174 ; IR ( KBr, $\mathrm{cm}^{-1}$ )

: $3410-3380(\mathrm{OH}$ and $\mathrm{NH}), 1600(\mathrm{C}=\mathrm{N})$, 1310 and $1110\left(\mathrm{SO}_{2}\right)$, 'HNMR(DMSO-d $\left.)_{6}\right) \mathrm{S}: 1.7$ $\left(\mathrm{t}, 3 \mathrm{H}, \mathrm{CH}_{3}\right), 2.5\left(\mathrm{q}, 2 \mathrm{H}, \mathrm{CH}_{2}\right), 4(\mathrm{~S}, \mathrm{IH}, \mathrm{OH}), 4.5(\mathrm{~S}, 2 \mathrm{H}$, $\left.\mathrm{CH}_{2}, \mathrm{~N}-\mathrm{CH}_{4}\right), 7.8(\mathrm{~m}, 4 \mathrm{H}, \mathrm{A}, \mathrm{H}), 9.1(\mathrm{~S}, 1 \mathrm{H}, \mathrm{NH})$. MFC $_{11} \mathrm{H}_{13} \mathrm{~N}_{3} \mathrm{O}_{3} \mathrm{~S}$. El - HS: m/z $267\left(\mathrm{M}^{+}\right)$.

(10c) yellow (60\%) ,mp 205-207, IR ( $\mathrm{KBr}$, $\left.\mathrm{cm}^{-1}\right)$ 3400-3370 (OH, and $\left.\mathrm{NH}\right), 1630(\mathrm{C}=\mathrm{N}) 1320$ and $1120\left(\mathrm{SO}_{2}\right)$. MF $\left(\mathrm{C}_{16} \mathrm{H}_{13} \mathrm{~N}_{3} \mathrm{O}_{3} \mathrm{~S}\right)$.

El - MS : m/z $329\left(\mathrm{M}^{+}\right)$
6- chloro -1,2,4- triazino - (4,3-b) $(1,2)$ benzisolhiazole(11)

A mixture of compound $3(10 \mathrm{~m}$ mole), phosphorous oxychloride ( $20 \mathrm{~m}$ mole) and $\mathrm{PCl}_{5}$ (1qm) was refluxed on a steam bath for $3 \mathrm{~h}$.

Then poured slowly into ice - cold water. The solid that separated was washed several times with water, dried and recrystallized from benzene to give compound (11); brown (70\%) ,mp 105-107. $\mathrm{IR}\left(\mathrm{KBr}, \mathrm{cm}^{-1}\right), \gamma 1630(\mathrm{C}=\mathrm{N}), 1330$ and $1120\left(\mathrm{SO}_{2}\right)$ and no absorption for $\gamma \mathrm{CO}$ and $\gamma \mathrm{NH}$.

$$
\text { MF } \mathrm{C}_{9} \mathrm{H}_{6} \mathrm{~N}_{3} \mathrm{O}_{2} \mathrm{~S} \mathrm{C}_{1} \text {. El - MS' : m/z } 255\left(\mathrm{M}^{+}\right)
$$
and $257\left(\mathrm{M}^{+}+2\right)$.

\section{Formation of compounds ( $12 \mathrm{a}$ and $\mathrm{b}$ )}

A mixture of compound 11 (10m mole) was refluxed with urea and / or thio- urea ( $10 \mathrm{~m}$ mole ) in $(40 \mathrm{ml})$ sodium ethoxide for $3 \mathrm{~h}$, then cooled and poured into water. The solid that separated was dried and recrystallized from benzene - ethanol $(1: 1)$ to give $12 \mathrm{a}$ and for $12 \mathrm{~b}$.

Compound (12a) : yellow (72\%), mp 72 -74. IR ( $\left.\mathrm{KBr}, \mathrm{cm}^{-1}\right) 3350-3320(\mathrm{NH}), 1610(\mathrm{C}=\mathrm{N})$, 1310 and $1120\left(\mathrm{SO}_{2}\right)$. MF $\mathrm{C}_{10} \mathrm{H}_{9} \mathrm{~N}_{5} \mathrm{O}_{3} \mathrm{~S}_{2}$ El- MS: $\mathrm{m} / 2$ $279\left(\mathrm{M}^{+}\right)$.

Compound (12b) : yellowish (65\%), mp 235- 237. IR $\left(\mathrm{KBr}, \mathrm{cm}^{-1}\right) 3370$-3330 (NH), $1630(\mathrm{C}=\mathrm{N}), 1240$ $(\mathrm{C}=\mathrm{S}) 1310$ and $1120\left(\mathrm{SO}_{2}\right)$. 'HNMR(DMSO-d $\left.{ }_{6}\right)$ :<smiles>[R]c1ccc2c(c1)C(=O)N(CC)[SH]2O[Na]</smiles>

Scheme 1: 
$4.2\left(\mathrm{~S} 2 \mathrm{H}, \mathrm{CH}_{2}, \mathrm{~N}-\mathrm{CH}_{2}\right), 5.9\left(2 \mathrm{~S}, 2 \mathrm{H}, \mathrm{NH}_{2}\right) \quad$ Acetic anhydride - acetic acid (1: 1) was refluxed for $7.5(\mathrm{~m}, 4 \mathrm{H}, \mathrm{ArH}), 8.2(\mathrm{~S}, \mathrm{IH}, \mathrm{NH}) . \mathrm{MF} \mathrm{C} 10 \mathrm{H} 9 \mathrm{~N} 5 \mathrm{O} 2$. 3h. after concentrated, the solid that separated was S2. El - MS : $\mathrm{m} / \mathrm{z} 295\left(\mathrm{M}^{+}\right)$.

cooled and recrystallized from benzene - ethanol $(1: 1)$ to give $(13 a-c)$.

6- OXO -5- arylidene - 1,2,4-triazino - (4,3-b) (1,2)benzisothiazole (13a-c).

\section{Compound 13a yellow (75\%),mp 105-107.}

IR $\left(\mathrm{KBr}, \mathrm{cm}^{-1}\right) 3400-3360$ (br. NH, OH), and aromatic aldehyde $(10 \mathrm{~m}$ mole) namely Benz aldehyde , p- chloro- Benz aldehyde and anisaldehyde in $(40 \mathrm{ml})$. 1720 (CO), 1560,1480 (C=N, C=C), 1350 and 1150 $\left(\mathrm{SO}_{2}\right)$. MF $\mathrm{C}_{16} \mathrm{H}_{11} \mathrm{~N}_{3} \mathrm{O}_{3} \mathrm{~S}$. El $-\mathrm{MS}: \mathrm{m} / \mathrm{z} 325\left(\mathrm{M}^{+}\right)$.

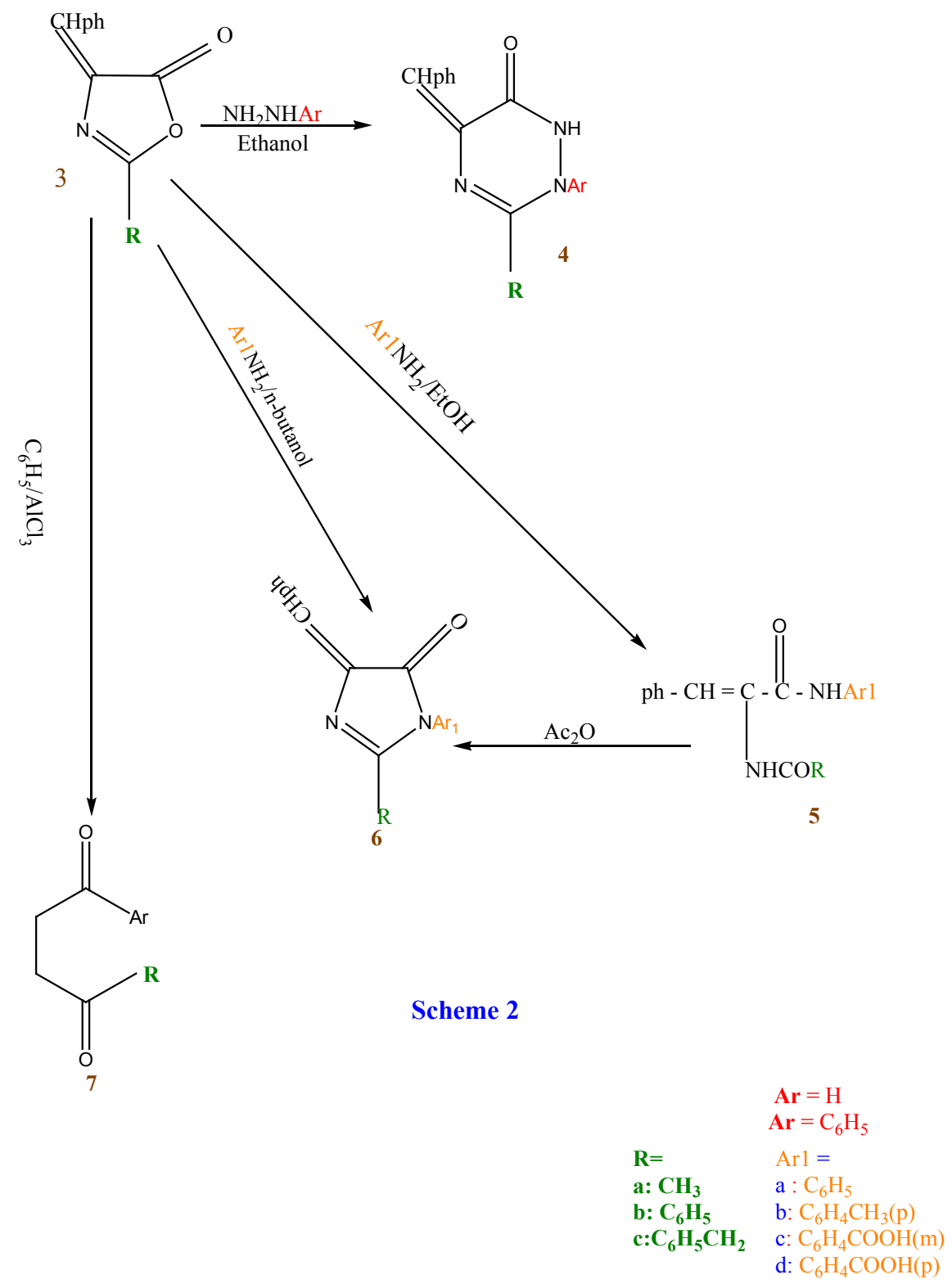

Scheme 2 : 
Compound 13b: yellow (75\%) ,mp 200 -202. IR ( $\left.\mathrm{KBr}, \mathrm{cm}^{-1}\right)$ 3420-3380(br. OH and $\left.\mathrm{NH}\right)$, 1700 (CO), 1580, 1460 ( $\mathrm{C}=\mathrm{N}, \mathrm{C}=\mathrm{C}$ ) , 1330 and $1120\left(\mathrm{SO}_{2}\right), \mathrm{MF} \mathrm{C}_{16} \mathrm{H}_{10} \mathrm{~N}_{3} \mathrm{O}_{3} \mathrm{SC}_{1}$. El- MS: $\mathrm{m} / \mathrm{x} 359$ $\left(\mathrm{M}^{+}\right)$AND 361 (2).

Compound 13c : ball yellow (65\%), mp 188-190. IR (KBR, cm$\left.{ }^{-1}\right)$ 3450- 3400 (br: $\mathrm{OH}$ and $\mathrm{NH}), 1720$ (CO) , 1610, $1450(\mathrm{C}=\mathrm{N}, \mathrm{C}=\mathrm{C}), 1300$ and $1110\left(\mathrm{SO}_{2}\right)$. MF $\mathrm{C}_{17} \mathrm{H}_{10} \mathrm{~N}_{3} \mathrm{O}_{4}$ S. El- MS: m/z 355(M+).

\section{Antimicrobial}

The newly synthesized compounds (3,4a-b, 5a-b, 5d, 5g, 6c, 7, 9, 10a, 11, 12b, and 13a) were tested for their in vitro growth inhibitory activity against a panel of standard strains of thelnstitute of fermentation of Osaka (IFO) namely; theGram-positive bacteria (Staphylococcus aureus IFO3060 and Bacillus subtilis IFO 3007), the Gramnegativebacteria (Escherichia coli IFO 3301 andProteus vulgaris IFO 3851.

The primary screening was carried out using the agar disc-diffusion method using MüllerHinton agar medium. ${ }^{24-28}$

\section{RESULTS AND DISCUSSION}

\section{Chemistry}

In the present investigation, saccharinyl acetic acid chloride ${ }^{9}$ reacted with glycine to give $\mathrm{N}$ - carboxymethylsaccharinylacetamide(2), which

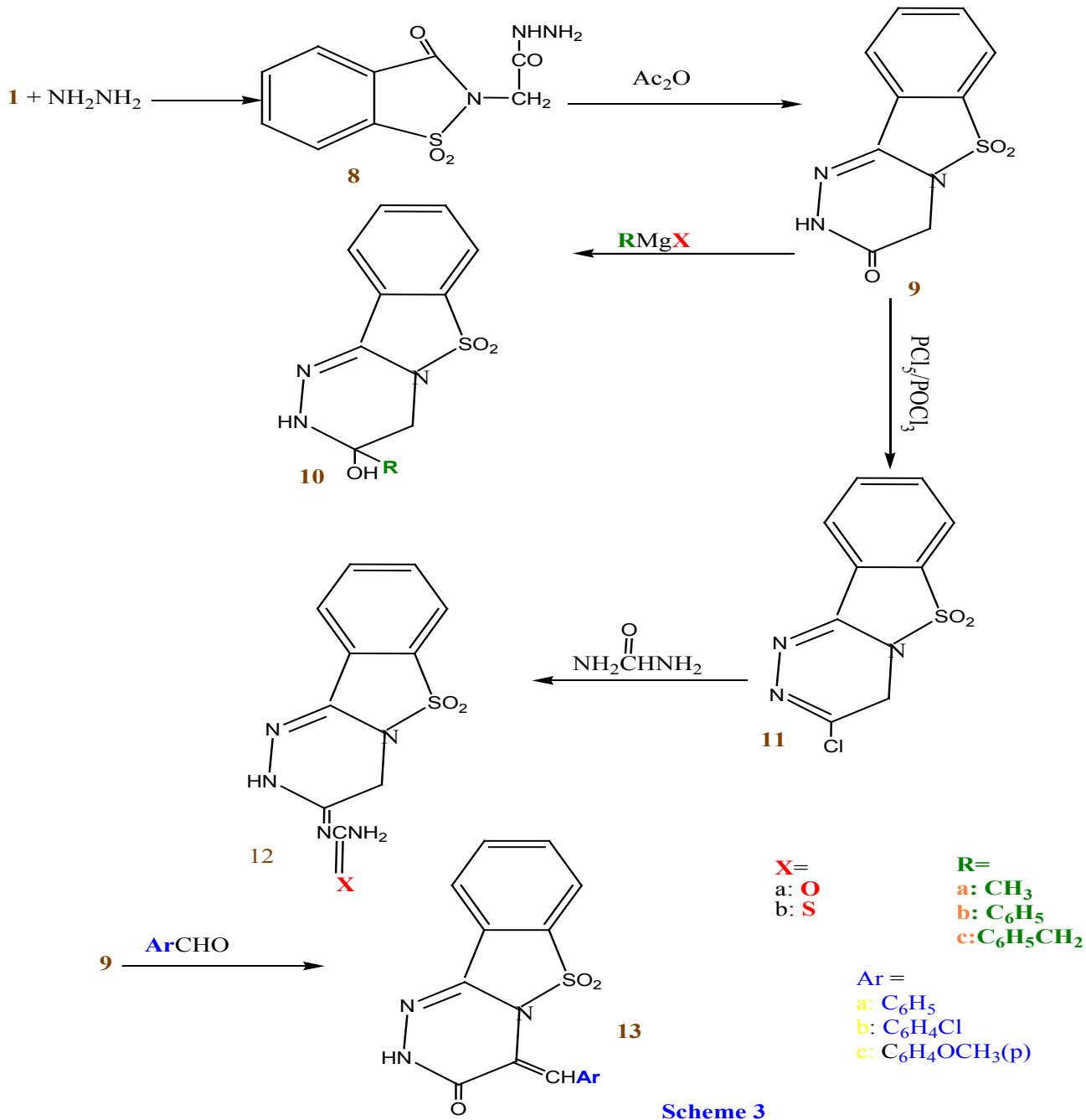

Scheme 3 : 
reacted with benzaldehyde in the presence of acetic anhydride and sodium acetate to give 4benzylidene-2-saccharinyl-methyl-1,3-oxzazole5 -one (3). Treatment of (3) with hydrazine and/ or phenylhydrazine in refluxing ethanol afforded 5-benzylidene-3-sacchrinyl-methyl-1, 2, 4 triazine-6one (4a) and/or 5-benzylidene-3-sacchrinyl-methyl2-N-phenyl-1, 2, 4- triazine-6-one (4b). Furthermore when compound(3) reacted with aromatic amines (aniline, $p$-toludine) and aminobenzoic acids ( $m$, and $p$-aminobezoic acid) in refluxing ethanol it gave cinnamimide derivatives ( $5 \mathrm{a}-\mathrm{d}$ ). But when the same reaction was carried out in $\mathrm{n}$-butanol it gave imidazolone derivatives (6a-d) which were also obtained by refluxing compounds (5a-d) in acetic anhydride. Moreover compound (3) undergoes acid catalyses ring opening reaction with dry benzene in presence of anhydrous $\mathrm{AlCl}_{3}$ (Fridel - Crafts reaction) to give saccharinyl - (N-benzoyl-methyl) acetamide (7), wherethe reaction product obtained via elimination of arylidene group ${ }^{23,24}$ On the other hand, when saccharinyl acetic acid hydrazide (8) ${ }^{9}$ was refluxed in acetic anhydride it cyclized to 6-oxo1,2,4-triazino-4,3- $\mathbf{b}^{1,2}$ benzisothiazole (9).

Also the behavior of compound (9) towards carbon nucleophiles (Grignard reagent) was investigated. Thus when compound (9) submitted to react with ethyl magnesium iodide, methyl magnesium chloride then refluxed in dry benzene, it afforded 6-alkyl-6-hydroxy-1,2,4-triazino-[4,3-b] $[1,2]$ benzisothiazole (10). Treatment of compound(9) with mixture of $\mathrm{POCl}_{3} / \mathrm{PCl}_{5}$ afforded 6-Chloro1,2,4-triazino[4,3-b][1,2]benzisothiazole (11) which reacted with urea and/or thiourea to give compound (12 $a$ and b) respectively.

Also when compound (9) was allowed to condense with aromatic aldehyde namely bezaldehyde, anisaldehyde, and p-chlorobezaldehde in the presence of pyridine in a mixture of acetic anhydride and acetic acid (1:1) it afforded 5-arylidene6-oxo-1,2,4-triazino-[4,3-b] benzisothiazole $(13 a-c)$.

\section{Antimicrobial Activity}

The antimicrobial activity (in vitro) of some synthesized compounds was determined against some bacteria and fungi using tetracycline and amphotericin $B$ as standard antimicrobial agents by using the agar diffusion method ${ }^{28},{ }^{29}$.

The obtained zones of inhibition were presented in table 1, which indicated that most of the synthesized derivatives have moderate to good antimicrobial activities.

\section{REFERENCES}

1. MagidAbou-Gharbia, John A.Moyer, UshaPatel, Micheal Webb, Guy Schiehser, Terrance Andree and J. Thomas Haskins, J. Med Chem.,1989,32, 1024

2. Richard Poul Dunlap, Neil Warren, Albert Mura and Dennis John Hlasta, (Sterling Drug, Inc.), PCT Int. Appl.,1990,549,15.US Appl.1989,347, 125,111pp.

3. Soon Kyoung Kwon and Myoung Suk, Arch. Pharmacal Res.1992, 15, 251-255

4. Donald Josef Dumas, EP 165003 A2US 85-72 6452, 1985, Appl, 111pp.

5. M.M.H.Arrief, M.S Amine and A.M.F Eissa, Egypt. J.Chem.,1999,42(6), 563-571

6. "M.S. Amine and M.M.H.Arief, Indian J.Chem.,1998,37,135-138

7. M.M.H Arief, S.G Donia, M.H.Azab and M.G Zinhong, Egypt,J.Chem,1998,41(1-6), 257-

\section{6}

8. M.M.H.Arief, Phosphorous, Sulfur, and Silicon, 1997, 127, 159-165.

9. M.M.H.Arief, Phosphorous, Sulfur, and Silicon, 1996, 114, 129-134.

10. A.A Failli, Us 4859671, 1989, Chem, Abst, 1990,112,7717.

11. D.J. Hlasta, R.C.Desai, C.Subramanyam, E.P.Lodge, R.P. Dunlap, N.W.Boaz, A.J. Mura, and L.H. Latimer, Eur.Pat.Appl. EP542372A1, 1993, Chem. Abst.1994, 120(15), 191707,.

12. W.C.Groutas, N. Houser-Archield, L.S.Chong, R.Venkataraman, J.B. Epp, H.Hunag and J.J. McCLenahan, J.Med.Chem.1993,36,317881, Chem. Abst.1993, 119,225875

13. N-Terzioglu, A.Gursoy, Eur.J.Med. Chem,2003,38,781

14. G.Kolavi, V.Hegde, I.Khan, P.Gaded, Bioorg. 
Med.Chem.2006, 14, 3069.

15. A.K. Gaded, C.S.Mohajanshetti, S.NimalKar, Eur.J.Med.Chem,2000,35, 853

16. M.Kidwai, P.Mothsra, Tetrahedron Lett.2006,47, 5029

17. C.S. Andotra, T.C. Langer, A. Kotha, J. Indian Chem. Soc.

18. S.A. Siddiqui, Tetrahedron Lett,2005,61, 3539

19. H.C. Kan, Tetrahedron Lett.2007,63, 1644

20. D. Luca, L.Curr, J. Med. Chem,2006, 13, 1

21. Adel A.H. Abdel-Rahman, shafey G. Donia, A.A.F.Wasfy, A.A. Aly and AmaalY. El-Gazzar, Der Pharma Chemica,2013, 5(1),196-204

22. R. Cruickshank, J.P. Dugui, B.P. Marion, R.H.A Swain, Medicinal Microbiology $12^{\text {th }}$ Edn. Churchill London,(1975), 196.

23. Murray PR, Baron EJ, Pfaller MA, TenoverFC, Yolken RH. Am. Soc. Micobiol.,Washington D.C. 1995
24. Atlas RM. Handbook of MicrobiologicalMedia. London: CRC Press 2004, 1226

25. Villanova PA. National Committee for Clinical Laboratory Standards (NCCLS)Approved standard document M-7A,1985

26. Sandven P, Lassen. Importance of Selective Media for Recovery of Yeasts from Clinical Specimens. J. Journal of clinical microbiology, 1999, 37(11),3731-3732

27. Magda H. Abdellattif, Mai. M. Helmy, Hany A. Eldeab, IJAPBC - 2014,3(4),

28. Rishiramparajuli, janmajoybanerjee, and hemantakhanal.oriental journal of chemistry,2015,31,(4).2099-2106

29. Reza moradivalikboni, zabialahheidarnezha d,fatemehheidarnezhad, yuldashboyhozhib oevandrahmanrahmanov, oriental journal of chemistry,2014, 30(1),391-394.

30. ChalakAzimi, Farhad Sepehraddin and HelalTahazadeh, ORIENTAL journal of chemistry,2013,29(4),1443-1448. 